\title{
Fast Direct Solution Algorithm for Electromagnetic Scattering from 3D Planar and Quasi-Planar Geometries ${ }^{\dagger}$
}

\author{
LEVENT GÜREL* \\ Dept. of Electrical \& Electr. Eng. \\ BILKENT UNIVERSITY \\ BILKENT, ANKARA, TURKeY \\ (1gurel@ee.bilkent.edu.tr)
}

\author{
WeNg Cho Chew \\ CTR. For COMPUTATIONAL Electromagnetics \\ DePt. OF EleCTRICAL \& COMPUTER ENG. \\ UNIVERSTTY OF ILLINOIS, URBANA, IL 61801 \\ (w-chewQuiuc.edu)
}

\section{Introduction}

Solutions of electromagnetic scattering problems involving 3D planar (Fig. 1) and quasiplanar [Fig. 4(a)] geometries in homogeneous and layered [Fig. 4(b)] media are of great interest due to the existence of a multitude of useful applications, such as frequency selective surfaces (FSSs), printed circuit boards (PCBs), microstrip structures, monolithic microwave integrated circuits (MMICs), and phased-array antennas, to name a few. Although several different techniques $[1-3]$ existed for the solution of these prob-
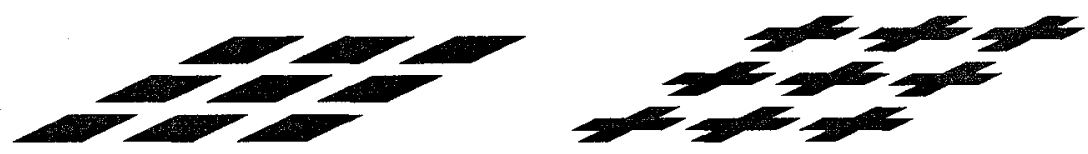

Figure 1: Planar geometries in a homogeneous medium.

lems, the need to solve larger problems with limited computational resources recently sparked the successful development of numerous new fast solvers [4-10]. However, no method can be expected to solve all classes of problems. For instance, the iterative solvers [4-6], which are perfectly suited for large problems, perform poorly for nearresonant structures. Some techniques are limited to $2 \mathrm{D}$ geometries $[7,8]$, whereas some others are limited homogeneous-medium problems [9,10]. Development of a new noniterative method and its application to planar geometries in homogeneous media will be presented in this paper. The method can easily and naturally be extended to the cases of quasi-planar structures and/or layered-media problems as will be discussed in Section 5 .

\footnotetext{
†This work was supported in part by NATO's Scientific Affairs Division in the framework of the Science for Stability Programme and in part by the Scientific and Technical Research Council of Turkey (TUBITAK) under contract EEEAG-163.
}

0-7803-4178-3/97/\$10.00 (C) 1997 IEEE 


\section{Fast Direct Algorithm Based on the Steepest Descent Path (FDA/SDP)}

The FDA/SDP takes advantage of the fact that the induced currents (i.e., basis and testing functions) on planar and quasi-planar geometries interact with each other within a very limited solid angle. Thus, all the degrees of freedom that are required to solve a "truly 3D" geometry are not required for a planar or a quasi-planar geometry, and this situation can be exploited to develop efficient solution algorithms. The FDA/SDP achieves its efficiency by essentially converting a 3D planar geometry to a "quasi-2D" geometry and then employing a fast $2 \mathrm{D}$ solver to efficiently solve this resulting "quasi2D" problem (Fig. 2). Assuming that the planar geometry is placed on the $x-y$ plane,

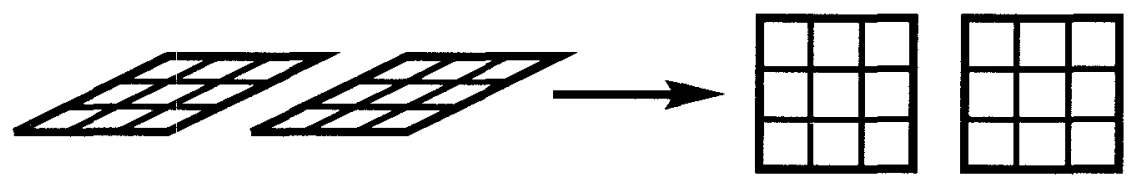

\section{D Problem}

\section{D Problem}

Figure 2: Perceiving a 3D planar object as a quasi-2D geometry.

tangential components of the electric field on the same plane are given by

$$
\left[\begin{array}{l}
E_{x}(\boldsymbol{\rho}) \\
E_{y}(\boldsymbol{\rho})
\end{array}\right]=i \omega \mu \int d \boldsymbol{\rho}^{\prime}\left[\overline{\mathbf{I}}+\frac{\nabla_{s}^{\prime} \nabla_{s}^{\prime}}{k^{2}}\right] \cdot g\left(\boldsymbol{\rho}, \boldsymbol{\rho}^{\prime}\right)\left[\begin{array}{l}
J_{x}\left(\boldsymbol{\rho}^{\prime}\right) \\
J_{y}\left(\boldsymbol{\rho}^{\prime}\right)
\end{array}\right]
$$

where $\boldsymbol{\rho}=\hat{x} x+\hat{y} y$ and $\boldsymbol{\rho}^{\prime}$ are arbitrary position vectors on the $x-y$ plane, $d \boldsymbol{\rho}^{\prime}=d x^{\prime} d y^{\prime}$, and $\nabla_{s}^{\prime}=\hat{x} \partial_{x^{\prime}}+\hat{y} \partial_{y^{\prime}}$. In the above, $g\left(\boldsymbol{\rho}, \boldsymbol{\rho}^{\prime}\right)$ is the 3D scalar Green's function restricted to the in-plane interactions and can be expressed in terms of the 2D Green's function using the identity [11]

$$
\begin{aligned}
g\left(\boldsymbol{\rho}, \boldsymbol{\rho}^{\prime}\right)=\frac{e^{i k\left|\boldsymbol{\rho}-\boldsymbol{\rho}^{\prime}\right|}}{4 \pi\left|\boldsymbol{\rho}-\boldsymbol{\rho}^{\prime}\right|} & =\frac{i}{8 \pi} \int_{-\infty}^{\infty} d k_{\rho} \frac{k_{\rho}}{k_{z}} H_{0}^{(1)}\left(k\left|\boldsymbol{\rho}-\boldsymbol{\rho}^{\prime}\right|\right) \\
& =\frac{k}{2 \pi} \int_{0}^{\infty} d s \frac{1+i s^{2}}{\sqrt{s^{2}-i 2}} H_{0}^{(1)}\left[k\left|\boldsymbol{\rho}-\boldsymbol{\rho}^{\prime}\right|\left(1+i s^{2}\right)\right]
\end{aligned}
$$

Equation (3) is obtained by deforming the path of integration in Eq. (2) to the steepest descent path (SDP), where the integrand is rapidly decaying. The SDP integral in Eq. (3) can be numerically evaluated by sampling the integrand at a set of appropriately chosen points $s_{m}$ with associated weights $w_{m}$ :

$$
\frac{e^{i k\left|\boldsymbol{\rho}-\boldsymbol{\rho}^{\prime}\right|}}{4 \pi\left|\boldsymbol{\rho}-\boldsymbol{\rho}^{\prime}\right|}=\frac{k}{2 \pi} \sum_{m} w_{m} \frac{1+i s_{m}^{2}}{\sqrt{s_{m}^{2}-i 2}} H_{0}^{(1)}\left[k\left|\boldsymbol{\rho}-\boldsymbol{\rho}^{\prime}\right|\left(1+i s_{m}^{2}\right)\right] .
$$

Thus, the 3D Green's function for this problem can be expressed as a sum of several 2D Green's functions. At this point, the problem can be solved using any $2 \mathrm{D}$ solver that has less than $O\left(N^{3}\right)$ computational complexity such as the RTMA [9], the RATMA [10], or the NEPAI, [12]. In this work, the RATMA will be used to solve the "quasi-2D" problem. 


\section{Numerical Integration}

Let $D_{\min }$ and $D_{\max }$ denote the smallest feature size and the largest dimension, respectively, of the "quasi-2D" problem such that the two may be different by several orders of magnitude. In order to solve the "quasi-2D" problem using the RATMA, the integrals

$$
\begin{array}{r}
\frac{e^{i k D_{\min }}}{4 \pi D_{\min }}+\ldots+\frac{e^{i k D_{\max }}}{4 \pi D_{\max }}=\frac{k}{2 \pi}\left\{\int_{0}^{\infty} d s \frac{1+i s^{2}}{\sqrt{s^{2}-i 2}} H_{0}^{(1)}\left[k D_{\min }\left(1+i s^{2}\right)\right]+\ldots+\right. \\
\left.\int_{0}^{\infty} d s \frac{1+i s^{2}}{\sqrt{s^{2}-i 2}} H_{0}^{(1)}\left[k D_{\max }\left(1+i s^{2}\right)\right]\right\}
\end{array}
$$

have to be evaluated by sampling their integrands at the same set of points $s_{m}$ to obtain the same accuracy for all of the integrals. Although the decay rates of the integrands can be very different as depicted in Fig. 3(a), an integration rule can be developed such that all of the above integrals can be computed using the same set of sampling points. The number of sampling points can be bounded by $O\left(\log \left(D_{\max } / D_{\min }\right)\right)$. Figure 3(b) shows the number of sampling points required to numerically compute the integrals of Eq. (5) for different levels of accuracy.

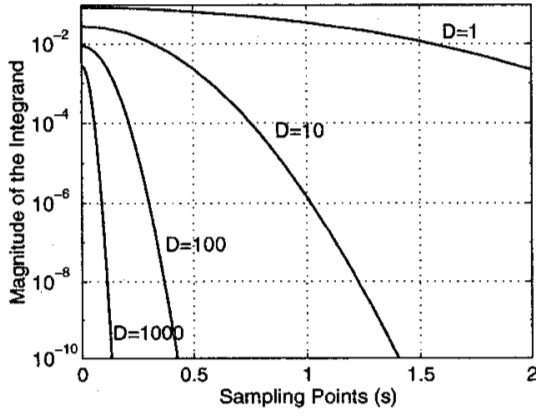

(a)



(b)

Figure 3: (a) Magnitude of the integrands corresponding to various feature sizes and distances $(D)$ in the problem, (b) number of sampling points required to obtain 3 to 6 correct significant digits (CSDs) in the computation of all integrals with different $D_{\max }$.

\section{Computational Complexity}

A careful analysis shows that the RATMA has $O\left(N P^{2}\right)$ computational complexity and $O\left(P^{2}\right)$ memory requirement [10], where $P$ is $O(\sqrt{N} \log \sqrt{N})$ for the dense "quasi-2D" problems considered in this work. Thus, the FDA/SDP has $O\left(N^{2} \log ^{2} N\right)$ computational complexity and $O\left(N \log ^{2} N\right)$ memory requirement. 


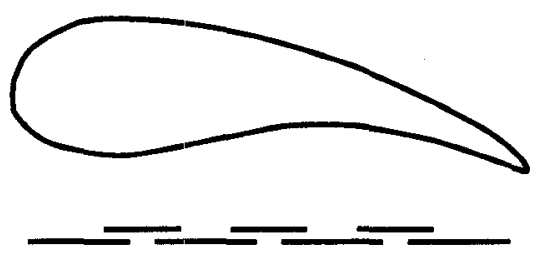

(a)

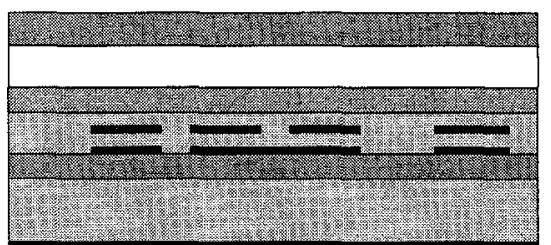

(b)

Figure 4: Cross-sectional views of (a) two quasi-planar geometries, and (b) a planar or quasi-planar geometry in a layered medium.

\section{Extensions}

The FDA/SDP can be extended from planar to quasi-planar structures, as shown in Fig. 4(a), where the geometry is not strictly planar, however, the size of the geometry in one dimension is much smaller than the other two dimensions. Furthermore, extension from homogeneous-media problems to layered-media problems [Fig. 4(b)] is also straightforward since the spectral-domain representation of the Green's function in Eq. (2) exists for layered media.

\section{References}

[1] T. Itoh, "Spectral domain immittance approach for dispersion characteristics of generalized transmission lines," IEEE Trans. Microwave Theory Tech., vol. MTT-28, pp. 733-736, July 1980.

[2] W. C. Chew and L. Gürel, "Reflection and transmission operators for strips or disks embedded in homogeneous and layered media," IEEE Trans. Microwave Theory Tech., vol. MTT-36, pp. 14881497 , Nov. 1.988

[3] L. Gürel and W. C. Chew, "Recursive algorithms for calculating the scattering from $N$ strips or patches," IEEE Trans. Antennas Propagat., vol. AP-38, pp. 507-515, Apr. 1990.

[4] R. Coifman, V. Rokhlin, and S. Wandzura, "The fast multipole method for the wave equation: a pedestrian prescription," IEEE Antennas Propagat. Mag., vol. 35, no. 3, pp. 7-12, June 1993.

[5] L. Gürel and M. I. Aksun, "Electromagnetic scattering solution of conducting strips in layered media using the fast multipole method," IEEE Microwave and Guided Wave Lett., vol. 6, no. 8, pp. 277-279, Aug. 1996.

[6] E. Michielssen and W. C. Chew, "Fast steepest descent path algorithm for analyzing scattering from two-dimensional objects," Radio Sci., vol. 31, no. 5, pp. 1215-1224, Sept.-Oct. 1996.

[7] W. C. Chew and C. Lu, "A recursive algorithm to compute the wave-scattering solution of a finite-strip array using an efficient plane-wave basis," Microwave Opt. Tech. Lett., vol. 5, no. 3, pp. 146-148, Mar. 1992.

[8] C. Lu and W. C. Chew, "Electromagnetic scattering of finite strip array on a dielectric slab," IEEE Trans. Microwave Theory Tech., vol. MTT-41, no. 1, pp. 97-100, Jan. 1993.

[9] L. Gürel and W. C. Chew, "A recursive T-matrix algorithm for strips and patches," Radio Science, vol. 27, pp. 387-401, May-June 1992.

[10] L. Gürel and W. C. Chew, "Recursive T-matrix algorithms with reduced complexities for scattering from three-dimensional patch geometries," IEEE Trans. Antennas Propagat., vol. AP-41, pp. 91-99, Jan. 1993.

[11] W. C. Chew, Waves and Fields in Inhomogeneous Media. Piscataway, NJ: IEEE Press, 1994.

[12] W. C. Chew and C. Lu, "The use of Huygens' equivalence principle for solving the volume integral equation of scattering," IEEE Trans. Antennas Propagat., vol. AP-41, no. 7, pp. 897-904, July 1993. 